\title{
Muon implantation experiments in films: Obtaining depth-resolved information
}

Cite as: Rev. Sci. Instrum. 91, 023906 (2020); https://doi.org/10.1063/1.5126529

Submitted: 04 September 2019 . Accepted: 30 January 2020 . Published Online: 13 February 2020

(D) A. F. A. Simões, (D) H. V. Alberto, (iD R. C. Vilão, (D) J. M. Gil, (D) J. M. V. Cunha, (D) M. A. Curado, (D) P. M. P. Salomé, (D. Trokscha, A. Suter, and (iD) Z. Salman

\section{ARTICLES YOU MAY BE INTERESTED IN}

Probing the solid-liquid interface with tender $\mathrm{x}$ rays: $\mathrm{A}$ new ambient-pressure $\mathrm{x}$-ray photoelectron spectroscopy endstation at the Swiss Light Source

Review of Scientific Instruments 91, 023103 (2020); https://doi.org/10.1063/1.5128600

Optical spectroscopy of muon/hydrogen defects in $6 \mathrm{H}-\mathrm{SiC}$

Journal of Applied Physics 127, 095702 (2020); https://doi.org/10.1063/1.5140733

Computational prediction of muon stopping sites: A novel take on the unperturbed electrostatic potential method

The Journal of Chemical Physics 153, 044111 (2020); https://doi.org/10.1063/5.0012381

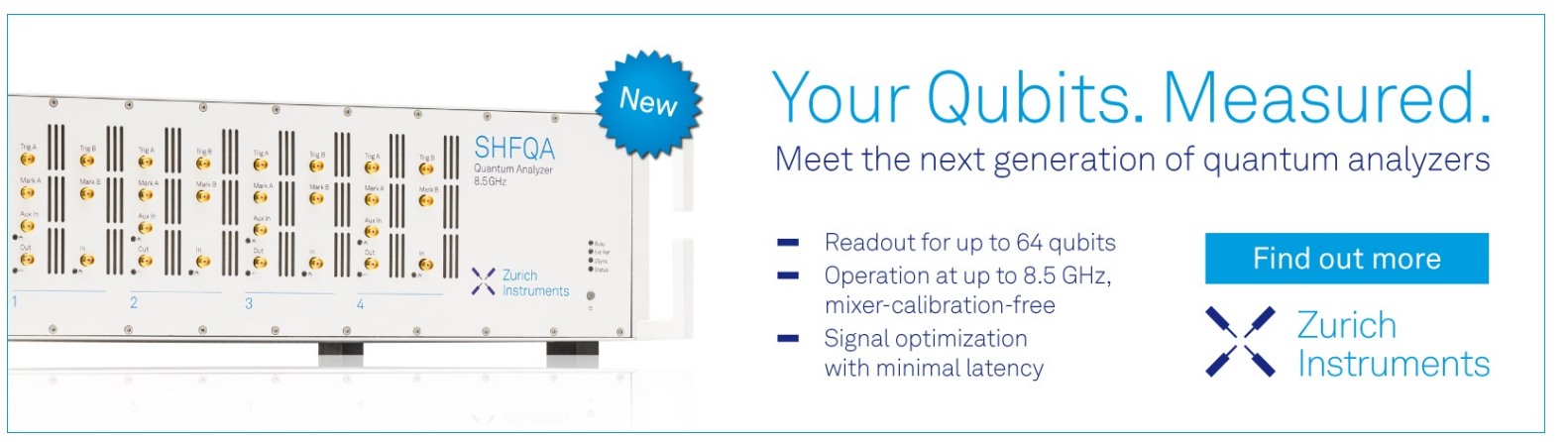




\title{
Muon implantation experiments in films: Obtaining depth-resolved information
}

\author{
Cite as: Rev. Sci. Instrum. 91, 023906 (2020); doi: 10.1063/1.5126529 \\ Submitted: 4 September 2019 - Accepted: 30 January 2020 • \\ Published Online: 13 February 2020
}
A. F. A. Simões,
H. V. Alberto,
R. C. Vilão,
J. M. Gil,
J. M. V. Cunha,
M. A. Curado,
P. M. P. Salomé,
T. Prokscha,
A. Suter, ${ }^{5}$ and Z. Salman

\begin{abstract}
AFFILIATIONS
${ }^{1}$ CFisUC, Department of Physics, University of Coimbra, R. Larga, P-3004-516 Coimbra, Portugal

${ }^{2}$ International Iberian Nanotechnology Laboratory, 4715-330 Braga, Portugal

${ }^{3}$ I3N, University of Aveiro, 3810-193 Aveiro, Portugal

4Department of Physics, University of Aveiro, 3810-193 Aveiro, Portugal

${ }^{5}$ Laboratory for Muon Spin Spectroscopy, Paul Scherrer Institute, CH-5232 Villigen PSI, Switzerland
\end{abstract}

a) Author to whom correspondence should be addressed: lena@uc.pt

\begin{abstract}
Implanted positive muons with low energies (in the range $1-30 \mathrm{keV}$ ) are extremely useful local probes in the study of thin films and multi-layer structures. The average muon stopping depth, typically in the order of tens of nanometers, is a function of the muon implantation energy and of the density of the material, but the stopping range extends over a broad region, which is also in the order of tens of nanometers. Therefore, an adequate simulation procedure is required in order to extract the depth dependence of the experimental parameters. Here, we present a method to extract depth-resolved information from the implantation energy dependence of the experimental parameters in a low-energy muon spin spectroscopy experiment. The method and corresponding results are exemplified for a semiconductor film, $\mathrm{Cu}(\mathrm{In}, \mathrm{Ga}) \mathrm{Se} \mathrm{e}_{2}, \mathrm{covered}$ with a thin layer of $\mathrm{Al}_{2} \mathrm{O}_{3}$, but can be applied to any heterostructure studied with low-energy muons. It is shown that if an effect is present in the experimental data, this method is an important tool to identify its location and depth extent.
\end{abstract}

Published under license by AIP Publishing. https://doi.org/10.1063/1.5126529

\section{INTRODUCTION}

In muon spin spectroscopy $(\mu \mathrm{SR})$ research, implanted positive muons, fully polarized, are used as local probes in a large variety of materials, ranging from superconductors, magnets, semiconductors, insulators, and metals to polymers and organic materials. ${ }^{1-3}$ The muon is a short-lived particle with an average lifetime of $2.2 \mu \mathrm{s}$. Once implanted, muons thermalize and end up decaying into positrons. The emitted positrons are detected outside the material, and their direction of emission holds information on the muon spin at the moment of decay. Therefore, the outcome of a $\mu$ SR experiment is the time evolution of the muon spin polarization, which is used to investigate the local environment of the muon.

In a conventional $\mu \mathrm{SR}$ experiment, the incoming muons have an energy of about $4 \mathrm{MeV}$. In a so-called slow-muon experiment, muons are produced with an energy chosen in the range $1-30 \mathrm{keV}$, allowing a control of the average implantation depth in the nanometer range, ${ }^{3,4}$ as illustrated in Fig. 1. This is achieved only in the Low-Energy Muon (LEM) Facility, at the Paul Scherrer Institute (PSI) in Switzerland, and constitutes an extremely useful tool for probing thin films and heterostructures.

The muon stopping distribution depends on the muon implantation energy and on the material density and composition, which can be simulated by Monte Carlo calculations using the Trim. SP code, ${ }^{4,5}$ as exemplified in Fig. 2. It is clear from the simulations that the width of the stopping distribution is of the order of tens of nanometers, being proportional to the implantation energy. Therefore, the depth resolution is not sufficient to directly extract the $\mu \mathrm{SR}$ parameters as a function of depth. This problem has been solved for superconductors, ${ }^{6,7}$ where the physical parameter that causes the effect is a magnetic field that varies with depth. Since there is a simple relation between the angular frequency of the $\mu \mathrm{SR}$ signal and the internal magnetic field of the material, a depth dependence of the magnetic field can be extracted from the $\mu$ SR data using 


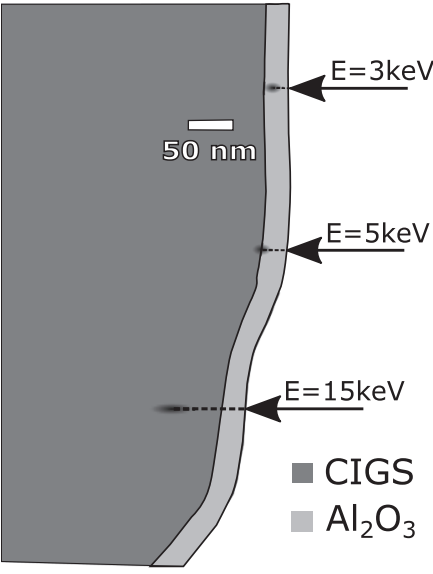

(a)

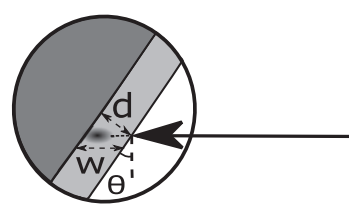

$\mathrm{w}=\mathrm{d} / \cos \theta$
FIG. 1. (a) Representation of the different implantation depths achieved with different muon implantation energies for an $\mathrm{Al}_{2} \mathrm{O}_{3} / \mathrm{CIGS}$ heterostructure. The drawing is based on a transmission electron microscopy picture. (b) Detail showing the effect of the roughness of the film surface: the effective thickness of the surface layer seen by the muon probe, $w$, is larger than the nominal thickness, $d$, and depends on the average value of the inclination of the film surface relative to the direction of the muon momentum.

one of two different approaches: (i) assuming a functional depth dependence of the magnetic field ${ }^{6}$ (an exponential shape is predicted in the so-called London limit, valid for a large class of superconductors) and (ii) calculating a magnetic field distribution as a function of implantation energy from the data and combining it with the muon stopping distribution as a function of depth and energy. This latter approach has the important advantage of being model

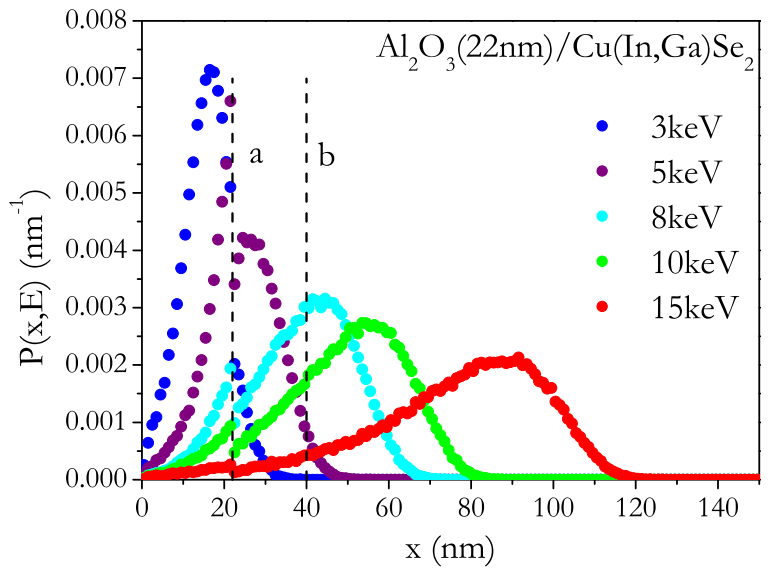

FIG. 2. Monte Carlo simulations of the probability per unit length, $P(x, E)$, that a muon implanted with energy $E$ stops at a depth $x$ in an $\mathrm{Al}_{2} \mathrm{O}_{3} / \mathrm{CIGS}$ junction, assuming a $22 \mathrm{~nm}$ thick $\mathrm{Al}_{2} \mathrm{O}_{3}$ layer and an average CIGS composition of $\mathrm{Cu}_{0.87} \mathrm{In}_{0.61} \mathrm{Ga}_{0.39} \mathrm{Se}_{2}$. The different curves correspond to different muon implantation energies. Two positions, $a=22 \mathrm{~nm}$ and $b=40 \mathrm{~nm}$, are marked as an example. For a given energy $E$, integrating the curve in the ranges $0<x<a, a<x<b$, and $b<x<\infty$ yields the corresponding probabilities $p_{0 a}(E), p_{a b}(E)$, and $p_{b \infty}(E)$ that the muons stop in each of the selected regions. independent but still requires previous knowledge of the functional relation between the measured $\mu \mathrm{SR}$ parameter and the underlying physical parameter that causes the effect. Another example is the recent determination of the hole carrier concentration profile at the surface of $\mathrm{Ge}$, where a linear relation between the muon depolarization rate and the hole concentration was assumed. ${ }^{8} \mathrm{~A}$ functional relation between the $\mu \mathrm{SR}$ parameter and the underlying physical parameter is not known in advance if the observed effect is a change of the amplitude of the $\mu \mathrm{SR}$ signal with the muon implantation energy, as often observed in semiconductors and insulators. In some of these cases, however, obtaining the depth dependence of the $\mu \mathrm{SR}$ parameter may be crucial for the interpretation of the data, namely, for phenomena occurring near interfaces in multi-layered structures.

In this work, we propose a method, based on simple and reasonable assumptions, to simulate the depth dependence of a given $\mu \mathrm{SR}$ parameter based on the experimentally observed energy dependence of the same parameter. We exemplify the method for a semiconductor film $\mathrm{Cu}(\mathrm{In}, \mathrm{Ga}) \mathrm{Se}_{2}$ (CIGS) covered with a thin layer $(22 \mathrm{~nm})$ of $\mathrm{Al}_{2} \mathrm{O}_{3}$. This sample is currently being investigated within a project aiming at characterizing the role of the dielectric oxide $\mathrm{Al}_{2} \mathrm{O}_{3}$ in the passivation of defects at the surface of the semiconductor CIGS, a material used in solar cell devices as an absorber. It has been shown that slow muons can be used as a probe of defect regions often present at the surface/interface of semiconductor and insulator materials, complementing the evidence obtained by other techniques that do not provide spatially resolved information. ${ }^{9,10}$ However, in order to quantify the information provided by the muon probe, a new method of analysis of the data needs to be developed.

The method developed in this work is, however, not limited to the choice of materials and can be applied to any insulator or semiconductor heterostructure. It can also be easily extended to any sample used in a low-energy muon experiment. The authors will provide the corresponding software code to anyone interested in it. The TRIMP.SP code is available from the LEM group.

\section{EXPERIMENTAL DATA}

The $\mu \mathrm{SR}$ measurements were performed at the $\mu \mathrm{E} 4$ beam line ${ }^{11}$ of the Swiss Muon Source, Paul Scherrer Institute, Switzerland, using the low-energy muon (LEM) instrument. Positive muons were implanted in the presence of an external magnetic field $\mathrm{B}=10 \mathrm{mT}$, using transverse field (TF) geometry into an $\mathrm{Al}_{2} \mathrm{O}_{3} / \mathrm{Cu}(\mathrm{In}, \mathrm{Ga}) \mathrm{Se}_{2}$ heterostructure, at the temperature $\mathrm{T}=40 \mathrm{~K}$. The muon implantation energy was tuned in the $3-15 \mathrm{keV}$ range in order to perform depth dependent studies.

The sample was produced in the Ångström Solar Center, Uppsala University, Sweden, using a co-evaporation test pilot tool for mini-module production. The sample consisted of soda lime glass (SLG), $350 \mathrm{~nm}$ of DC-sputtered Mo, and CIGS, where the latter was deposited at a substrate temperature of $530{ }^{\circ} \mathrm{C}$ with an average thickness of $1.94 \mu \mathrm{m}$. The average CIGS composition is $\mathrm{Cu}_{0.87} \mathrm{In}_{0.61} \mathrm{Ga}_{0.39} \mathrm{Se}_{2}$, within the region accessed by the muon. $\mathrm{Al}_{2} \mathrm{O}_{3}$ was deposited on top of CIGS by atomic layer deposition (ALD) at a substrate temperature of $100^{\circ} \mathrm{C}$ with trimethylaluminum (TMA) and water as precursors. 
The thickness of the $\mathrm{Al}_{2} \mathrm{O}_{3}$ layer was measured by Transmission Electron Microscopy (TEM) in the International Iberian Nanotechnology Laboratory (INL), yielding $22 \mathrm{~nm} \pm 4 \mathrm{~nm}$. The effective thickness of the $\mathrm{Al}_{2} \mathrm{O}_{3}$ layer seen by using the muon probe is widened by the inclination of the film surface relative to the direction of the muon beam, as depicted in Fig. 1(b). In order to obtain the effective thickness of the upper layer as seen by the muon probe, an average value of the surface inclination needs to be evaluated, using the area as a weighting factor. In the case of this film, a careful examination of the TEM images showed no significant widening of the $\mathrm{Al}_{2} \mathrm{O}_{3}$ thickness along the muon beam direction, and therefore, we will take $22 \mathrm{~nm} \pm 4 \mathrm{~nm}$ as the reference value for the $\mathrm{Al}_{2} \mathrm{O}_{3}$ layer thickness in this experiment.

We recall that the growth of the interface is often accompanied by interdiffusion phenomena creating defect layers that do not change the average composition significantly and that are largely invisible to most of the measuring methods (including TEM). TEM is, therefore, most adequate to obtain the width of the material layers. The muon probe is highly sensitive to the presence of defects, which have a non-negligible impact on the $\mu \mathrm{SR}$ signal and allow it to give essential microscopic information about the phenomena occurring at the interface.

Figure 3 shows a $\mu \mathrm{SR}$ time spectra of an $\mathrm{Al}_{2} \mathrm{O}_{3} / \mathrm{CIGS}$ sample at an implantation energy of $3 \mathrm{keV}$ and temperature $40 \mathrm{~K}$ in the presence of a transverse magnetic field of $10 \mathrm{mT}$. The complete set of data was described using an exponentially damped cosine at an angular frequency, which is close to the muon Larmor frequency. This signal corresponds to muons forming a state with no net atomic magnetic moment, that is, a diamagnetic state. The data were, therefore, fitted with a function of the following form:

$$
A(t)=A_{\text {dia }} \mathrm{e}^{-\lambda t} \cos (\omega t+\phi),
$$

where $A_{\text {dia }}$ is the diamagnetic signal amplitude, $\lambda$ is the exponential depolarization rate, $\omega$ is the angular frequency, and $\phi$ is the phase. It should be noted that the CIGS data are usually fitted using a

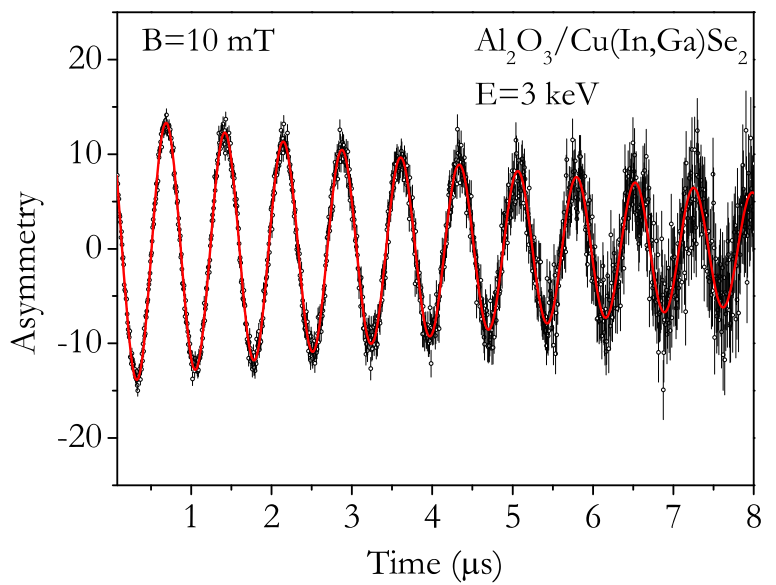

FIG. 3. $\mu \mathrm{SR}$ time spectrum in an $\mathrm{Al}_{2} \mathrm{O}_{3} / \mathrm{Cu}(\mathrm{In}, \mathrm{Ga}) \mathrm{Se}_{2}$ sample at $\mathrm{T}=40 \mathrm{~K}$ in transverse geometry (with an applied magnetic field $\mathrm{B}=10 \mathrm{mT}$ ) and a muon implantation energy of $3 \mathrm{keV}$. The red line is the result of fitting the data to an exponentially damped cosine function. gaussian damping, ${ }^{9,12-15}$ whereas the adequate relaxation function for $\mathrm{Al}_{2} \mathrm{O}_{3}$ is the exponential. ${ }^{16}$ Our slow-muon data do not allow for a precise determination of the shape of the relaxation, and an exponential relaxation function was chosen for the complete set of implantation energies. All the parameters $A_{\text {dia }}, \lambda, \omega$, and $\phi$ were used as free parameters in fitting the time dependent data. In this sample, the amplitude of the visible $\mu$ SR signal was always smaller than the expected maximum asymmetry, as obtained from a silver calibration at $200 \mathrm{~K}$. In other words, part of the total $\mu \mathrm{SR}$ signal is missing, a situation often found in materials for which the muons experience a strong interaction during their implantation stage. ${ }^{2,16-21}$ Therefore, the parameter $A_{\text {dia }}$ was converted to the corresponding fraction of the total $\mu \mathrm{SR}$ signal, $f_{\text {dia }}$, using the silver calibration and a sample size correction, to account for the fraction of muons landing in the sample plate.

The $\mu$ SR parameters $-f_{\text {dia }}, \lambda, \omega$, and $\phi$-as a function of the muon implantation energy, $E$, constitute the direct experimental data. The following method is proposed to infer the corresponding depth dependence of the same parameters.

\section{METHOD}

As discussed before, the muon stopping range inside the material extends over a broad region and the depth resolution of the experiment is not sufficient to directly convert the energy dependence of a given $\mu$ SR parameter-we will call it $f(E)$ - into a depth dependence, $f(x)$. The simplest approach to overcome this difficulty is to divide the sample into layers, assuming that the $f$ parameter is constant within each layer. In our case, it is reasonable to expect at least two layers: $0<x<a$ and $x>a$, where $a$ is the $\mathrm{Al}_{2} \mathrm{O}_{3}$ layer thickness. The constant value of $f$ would be a value typical for $\mathrm{Al}_{2} \mathrm{O}_{3}$ or $\mathrm{Cu}(\mathrm{In}, \mathrm{Ga}) \mathrm{Se}_{2}$ for the first or second layer, respectively. For such a two-layer system, it is reasonable to assume an abrupt change in $f$ between layers, considering both the composition change and the limited experimental depth resolution. However, some parameters may exhibit a change in $f$ within a given material, namely, close to the interface of the two materials, which requires creating an additional near-interface region for $f(x)$.

Let us consider a case where the data suggest that $f(x)$ has three main regions. The simplest approach is to assume that $f(x)$ is a steplike function given by

$$
f(x)=\left\{\begin{array}{llc}
f_{0 a} & \text { for } & 0 \leq x<a, \\
f_{a b} & \text { for } & a \leq x \leq b, \\
f_{b \infty} & \text { for } & x>b,
\end{array}\right.
$$

where $f_{0 a}, f_{a b}$, and $f_{b \infty}$ are the average values of the $\mu$ SR parameter $f$ in the ranges $0<x<a, a<x<b$, and $x>b$, respectively. Within this model, $f(E)$ is calculated as

$$
f(E)=p_{0 a}(E) f_{0 a}+p_{a b}(E) f_{a b}+p_{b \infty}(E) f_{b \infty},
$$

where $p_{0 a}(E), p_{a b}(E)$, and $p_{b \infty}(E)$ are the probabilities that a muon with energy $E$ stops in the respective depth range. The values of $f_{0 a}$, $f_{a b}, f_{b \infty}, a$, and $b$ are adjustable parameters used to obtain a function $f(E)$ that describes the experimental data.

The probability $p_{a b}(E)$ that the muon implanted with energy $E$ stops in the range $a<x<b$ can be calculated by numerical 
integration of the muon stopping probability per unit length, $P(x, E)$,

$$
p_{a b}(E)=\int_{a}^{b} P(x, E) d x,
$$

where $P(x, E)$ is obtained by Monte Carlo simulation, using the TRIM.SP code, ${ }^{4,5}$ as exemplified in Fig. 2. This simulation requires as input the average sample composition and layer width, which, as mentioned in Sec. II, were determined by the adequate sample characterization methods.

This model can be refined to allow a smooth transition between two different values of $f$ occurring in a finite region, starting at $x=b$ and ending at $x=c$. A sigmoidal shape was chosen to describe a smooth transition centered at $x_{0}=(b+c) / 2$ and with a slope determined by a parameter $d$. The following alternative to Eq. (2) was, therefore, considered:

$$
f(x)= \begin{cases}f_{0 a} & \text { for } 0 \leq x<a, \\ f_{a b} & \text { for } a \leq x<b, \\ f_{c \infty}+\frac{f_{a b}-f_{c \infty}}{1+\mathrm{e}^{\left(x-x_{0}\right) / d}} & \text { for } b \leq x \leq c, \\ f_{c \infty} & \text { for } x \geq c,\end{cases}
$$

where $x_{0}=(b+c) / 2$. Since a sigmoidal shape requires that $d \ll(c-b), d$ was defined as $d=(c-b) / 10$.

If $f(x)$ is given by Eq. (5), the adjustable parameters are $f_{0 a}, f_{a b}$, $f_{c \infty}, a, b$, and $c$. The corresponding function $f(E)$ becomes

$f(E)=p_{0 a}(E) f_{0 a}+p_{a b}(E) f_{a b}+\int_{b}^{c} P(x, E) f(x) d x+p_{c \infty}(E) f_{c \infty}$.

The procedure for the analysis is as follows: initial values for the adjustable parameters are inferred from a visual inspection of the experimental data. The function $f(E)$ is calculated using Eq. (3), and the experimental data points are fitted to the calculated $f(E)$ using the adjustable parameters. A MATLAB routine was developed to fit the experimental $\mu \mathrm{SR}$ parameters as a function of muon implantation energy, using this model. The routine calculates a theoretical $f(E)$ function as described above and then utilizes the program Fminuit $^{22}$ to fit this function to the experimental $f(E)$ data. Fminuit is a free fitting program that runs under Matlab and is based on the Minuit minimization package developed at CERN. The final fitting parameters are used to generate the depth dependent function, $f(x)$, corresponding to the best description of the experimental data.

As mentioned above, the function $f(x)$ was assumed, for simplicity, to be constant within each layer, but the method can be modified to allow a different functional dependence of $f(x)$ (e.g., linear, exponential, or other), especially if there is some physical model that suggests its use.

\section{RESULTS}

As mentioned in Sec. II, the $\mu$ SR signal in an $\mathrm{Al}_{2} \mathrm{O}_{3} /$ CIGS sample has a single component, and therefore, we have only four experimental parameters: diamagnetic fraction, $f_{\text {dia }}$, muon spin relaxation rate, $\lambda$, angular frequency, $\omega$, and initial phase, $\phi$. All these parameters exhibit changes with the muon implantation energy, except $\phi$ which will be ignored in the following discussion.

Figure 4 shows, at left, the muon spin relaxation, $\lambda$, and the local magnetic field, as a function of the muon implantation energy, $E$. The effective magnetic field at the muon position, $B_{\text {eff }}$, is calculated as $B_{\text {eff }}=\frac{\omega}{\gamma_{\mu}}$, where $\gamma_{\mu} / 2 \pi=1.355 \times 10^{8} \mathrm{~Hz} / \mathrm{T}$ is the muon gyromagnetic ratio. The data were analyzed assuming only two layers and an abrupt change in the parameter at the layer interface, as shown in Fig. 4, at right. The parameters obtained by fitting the data to the referred model are presented in Table I. The full curves obtained lead to a good description of the data, even with this simple model. Allowing a smooth transition between layers did not improve the fitting since the frontiers of the transition always converged to values that are equal within the uncertainties. It is important to note that the thickness of the first layer obtained by fitting the data is consistent with the experimental value of the thickness of the $\mathrm{Al}_{2} \mathrm{O}_{3}$ layer, as obtained from TEM measurements. Therefore, for both the muon relaxation rate and the local field at the muon site, the changes with depth are only due to a composition change. The exponential relaxation in the CIGS material is consistent with the value of the gaussian relaxation observed previously, ${ }^{9,12-15}$ after taking the difference in shape in consideration. The exponential relaxation observed for $\mathrm{Al}_{2} \mathrm{O}_{3}$ is consistent with the values reported in the literature. ${ }^{23,24}$

Concerning the effective magnetic field at the muon site, it is just the externally applied field in the case of $\mathrm{Al}_{2} \mathrm{O}_{3}$, whereas it is slightly higher in CIGS, indicating that the measured signal actually contains a paramagnetic component, which is also consistent with previous reports.
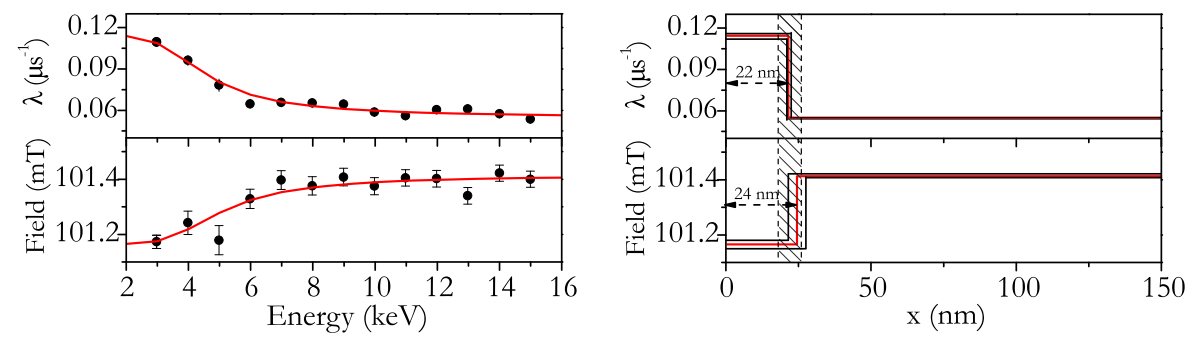

FIG. 4. Left: muon spin relaxation, $\lambda$, and local magnetic field at the muon position as a function of muon implantation energy at $40 \mathrm{~K}$. The red curve is the predicted behavior of the parameters assuming a depth dependence, as shown in the corresponding graph at right. Right: muon spin relaxation, $\lambda$, and local magnetic field as a function of sample depth, $x$. The functions $\lambda(x)$ and $B_{\text {eff }}(x)$ are assumed to have a step-like function with two regions. The functions are obtained by fitting the data at left and are represented by red lines. Those lines are at the center of an envelope that expresses the uncertainties in the parameters. The shaded area represents the interface region between $\mathrm{Al}_{2} \mathrm{O}_{3}$ and $\mathrm{CIGS}$ layers, as obtained from TEM measurements: $22 \mathrm{~nm} \pm 4 \mathrm{~nm}$. 
TABLE I. Fitting parameters and reduced $\chi^{2}$ obtained in the analysis of the relaxation rate $(\lambda)$ and effective magnetic field, $B_{\text {eff }}$, as a function of the muon implantation energy (Fig. 4).

\begin{tabular}{lcc}
\hline \hline & $\lambda\left(\mu \mathrm{s}^{-1}\right)$ & $B_{\text {eff }}(\mathrm{mT})$ \\
\hline $\mathrm{Al}_{2} \mathrm{O}_{3}$ & $0.114(2)$ & $10.117(3)$ \\
$\mathrm{CIGS}$ & $0.056(1)$ & $10.141(1)$ \\
1 st layer thickness $(\mathrm{nm})$ & $22(1)$ & $24(3)$ \\
$\chi_{\text {red }}^{2}$ & 1.4 & 1.1 \\
\hline \hline
\end{tabular}

If the same approach is applied to the diamagnetic fraction $f_{\text {dia, }}$, that is, assuming only two regions for $f_{\text {dia }}(x)$ and an abrupt change in the parameter at the interface, the description of the data is poor [Fig. 5(a)], with an associated $\chi_{\text {red }}^{2}=3.4$ (see Table II).

This suggests that an additional region should be considered for the $f_{\text {dia }}(x)$ function. It is important to note that the experimental data $f_{\text {dia }}(E)$ does not, by itself, give any clue where this third region is located (within $\mathrm{Al}_{2} \mathrm{O}_{3}$ or within CIGS, or in both) or about its spatial extent. Obtaining a depth-resolved information, i.e., $f_{\text {dia }}(x)$, is, therefore, crucial for the interpretation of the data. In Fig. 5(b), three regions were considered, assuming an abrupt transition between them, whereas in Fig. 5(c), the two transitions between regions were assumed to have a sigmoidal shape. The improvement in the fit quality clearly justifies the addition of a third region. In both (b) and (c) options, the final $f_{\text {dia }}(x)$ function clearly shows that the intermediate region is on the CIGS side, close to the $\mathrm{Al}_{2} \mathrm{O}_{3}$ interface. In option (c), the first smooth transition is, in fact, equivalent to an abrupt one since its limits are the same within the uncertainties. However, the transition within CIGS is well described by a smooth change which is probably closer to reality. However, the fit results presented in Table II indicate that this refinement does not lead to an improvement in the description of the data. It is also possible that in some cases, there is an overlap between the limits of two different regions separated by smooth transitions, especially if the regions are narrow. The present version of the program also includes other options such as a linear transition between regions that may be tried
TABLE II. Fitting parameters and reduced $\chi^{2}$ obtained in the analysis of the diamagnetic fraction as a function of the muon implantation energy depicted in Figs. 5(a)5(c). The case of four layers with abrupt transitions is also included. In (c), the center of the transition regions was used to measure the thickness of the layers; the parameter $d$ was $d_{1}=0.012(16) \mathrm{nm}$ and $d_{2}=4.6(4) \mathrm{nm}$ for the first and second transitions, respectively.

\begin{tabular}{lcccc}
\hline \hline & \multicolumn{4}{c}{ Diamagnetic fraction (\%) } \\
\cline { 2 - 5 } & (a) & (b) & (c) & 4 layers \\
\hline $\mathrm{Al}_{2} \mathrm{O}_{3}$ & $74.8(9)$ & $72.3(8)$ & $69(9)$ & $69(9)$ \\
$\mathrm{CIGS}$ (close to interface) & $84.2(3)$ & $82.1(3)$ & $81(1)$ & $80(2)$ \\
CIGS (intermediate bulk) & $\ldots$ & $\ldots$ & $\ldots$ & $84(2)$ \\
CIGS (bulk) & $84.2(3)$ & $86.0(5)$ & $85.4(2)$ & $86(1)$ \\
1st layer thickness (nm) & $31(4)$ & $22(1)$ & $18(2)$ & $16.1(1)$ \\
2nd layer thickness (nm) & $\ldots$ & $42(3)$ & $33(4)$ & $30(3)$ \\
3rd layer thickness (nm) & $\ldots$ & $\ldots$ & $\ldots$ & $30(3)$ \\
$\chi_{\text {red }}^{2}$ & 3.4 & 1.6 & 1.8 & 1.6 \\
\hline \hline
\end{tabular}

in such cases, but it is important to be aware that there is an intrinsic limitation in the depth resolution due to the width of the muon implantation profiles. It should also be noted that in model (c), the uncertainty in the diamagnetic fraction for the $\mathrm{Al}_{2} \mathrm{O}_{3}$ layer is very large (see Table II and Fig. 5). This is due to a correlation between the two parameters of the $\mathrm{Al}_{2} \mathrm{O}_{3}$ layer: the lower the layer thickness, the larger is the uncertainty in the fraction, and additional data points at low implantation energies are needed to define the initial slope of the function $f_{\text {dia }}(E)$. It is important to be aware, however, that for implantation energies below $3 \mathrm{keV}$, a significant fraction of the muons does not stop in the sample due to backscattering and the data must be carefully corrected for this experimental artifact. We have also checked adding a fourth layer with abrupt boundaries. The values for the diamagnetic fraction for the third and fourth layers are consistent within the uncertainties, making it difficult to distinguish from the three-layer solution with abrupt boundaries, which has a similar reduced $\chi^{2}$ to the four-layer solution.
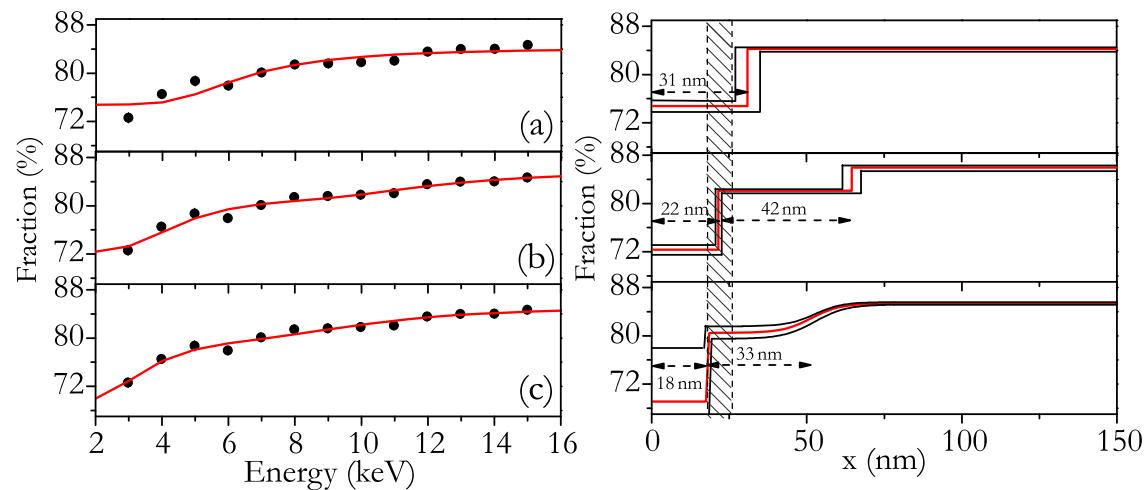

FIG. 5. Left: diamagnetic fraction as a function of muon implantation energy, $f_{\text {dia }}(E)$, at $40 \mathrm{~K}$. The red curves are the predicted behavior of the parameter assuming a depth dependence, $f_{\mathrm{dia}}(x)$, as shown in the corresponding graph at right. Right: diamagnetic fraction as a function of depth, $f_{\mathrm{dia}}(x)$, and the corresponding envelope, which expresses the uncertainties in the parameters. In (a), a one step function is assumed. In (b) and (c), three regions are considered, but in (c), a smooth change is allowed for $f_{\text {dia }}(x)$ in both transitions. The shaded area represents the interface region between $\mathrm{Al}_{2} \mathrm{O}_{3}$ and $\mathrm{CIGS}$ layers, as obtained from TEM measurements. 
Evidence for the presence of two regions within the CIGS material with different formation probabilities of the diamagnetic muon state was already reported in CdS/CIGS and ZnSnO/CIGS heterostructures and was attributed to a disordered region in CIGS, close to the interface with the other material. In the $\mathrm{Al}_{2} \mathrm{O}_{3} / \mathrm{CIGS}$ system, the effect is much less evident in the experimental $f_{\text {dia }}(E)$ data, when compared with the corresponding data in CdS/CIGS and $\mathrm{ZnSnO} / \mathrm{CIGS}$, but its presence can be revealed with a depth-resolved analysis.

In this case, the thickness of the first layer was found to be consistent with the thickness of the first material in the heterostructure, but this is not necessarily always the case. It is well known that in some heterostructures (namely, in CdS/CIGS ${ }^{25}$ ), there is a diffusion of ions between the two materials near the interface and the different $\mu$ SR parameters may have different sensitivities to this interlayer diffusion. This may lead to different functional dependences of the $\mu \mathrm{SR}$ parameters. For the $\mathrm{Al}_{2} \mathrm{O}_{3} / \mathrm{CIGS}$ system, however, no atomic intermixing is expected and the LEM data reproduce the structural measurements well within the uncertainties. Concerning the diamagnetic fraction, it is important to note that the change in the muon response within CIGS exists even in the absence of a top layer, that is, in a single CIGS film. ${ }^{9,26}$ The change in the diamagnetic fraction at the CIGS surface has been interpreted as due to a surface defect layer, whose presence is well documented in the literature. ${ }^{27-29}$ The width of this surface defect layer reduces to about half of its original value when a CdS layer is deposited on CIGS, ${ }^{26}$ whereas a much smaller reduction is observed when the top layer is $\mathrm{Al}_{2} \mathrm{O}_{3}$.

\section{CONCLUSIONS}

We present a method to extract the depth-resolved information for the muon probe in a low-energy muon experiment. The experimental parameters are obtained as a function of the muon implantation energy, but if the energy dependent data exhibit an effect, inferring the corresponding depth dependent function may be crucial to understand the contribution of each layer for the effect and its depth extent.

The method requires assuming a function for the depth dependence with variable parameters that are adjusted in order to describe the experimental energy dependent data. It is shown that the simple approach of dividing the sample in layers and assuming step functions for the depth dependence is a good approximation within the depth resolution of the muon probe since a refinement of the model did not lead to any significant improvement in the quality of the fit.

The method was exemplified for a semiconductor heterostructure but can be applied to any material. Although we present data for one sample only, we have used this method successfully and reproducibly for the analysis of heterostructure data obtained with LEM, either by reanalysis of previous data ${ }^{26}$ or novel data (to be published elsewhere). The results obtained using this method ${ }^{26}$ are fully consistent with the analysis of the same samples with complementary techniques ${ }^{25,30}$ but offer important additional information concerning the spatial extent of observed effects.

The method presented in this work is meant to be used in situations where the functional relation between the measured $\mu \mathrm{SR}$ parameters and the underlying physical parameters is not known, but it can be extended by including known functional relations.
Therefore, it constitutes a general tool for the analysis of slow-muon experiments.

\section{ACKNOWLEDGMENTS}

This work is based on experiments performed at the Swiss Muon Source $(\mathrm{S} \mu \mathrm{S})$, Paul Scherrer Institute, Villigen, Switzerland. This work was financially supported by the Portuguese National Budget through Fundação para a Ciência e Tecnologia/MCTES and the European Regional Development Fund (ERDF) through the Competitiveness and Internationalisation Operational Program (PT-COMPETE 2020), under Project Nos. UID/FIS/04564/2016 and PTDC/FISMAC/29696/2017 and Grant No. PD/BD/142780/2018.

\section{REFERENCES}

${ }^{1}$ P. J. C. King, R. de Renzi, S. P. Cottrell, A. D. Hillier, and S. F. J. Cox, "ISIS muons for materials and molecular science studies," Phys. Scr. 88, 068502 (2013).

${ }^{2}$ S. F. J. Cox, "Muonium as a model for interstitial hydrogen in the semiconducting and semimetallic elements,” Rep. Prog. Phys. 72, 116501 (2009).

${ }^{3}$ PSI, Internet page of the laboratory for muon spectroscopy of the Paul Scherrer Institute, 2019, https://www.psi.ch/lmu/; accessed 9 July 2019.

${ }^{4}$ E. Morenzoni, H. Glückler, T. Prokscha, R. Khasanov, H. Luetkens, M. Birke, E. Forgan, C. Niedermayer, and M. Pleines, "Implantation studies of keV positive muons in thin metallic layers," Nucl. Instrum. Methods Phys. Res., Sect. B 192, 254-266 (2002).

${ }^{\mathbf{5}}$ W. Eckstein, Computer Simulations of Ion-Solid Interactions (Springer, Berlin, Heidelberg, New York, 1991)

${ }^{6}$ R. F. Kiefl, M. D. Hossain, B. M. Wojek, S. R. Dunsiger, G. D. Morris, T. Prokscha, Z. Salman, J. Baglo, D. A. Bonn, R. Liang, W. N. Hardy, A. Suter, and E. Morenzoni, "Direct measurement of the London penetration depth in $\mathrm{YBa}_{2} \mathrm{Cu}_{3} \mathrm{O}_{6.92}$ using low-energy $\mu$ SR,” Phys. Rev. B 81, 180502 (2010).

${ }^{7}$ A. Suter, E. Morenzoni, N. Garifianov, R. Khasanov, E. Kirk, H. Luetkens, T. Prokscha, and M. Horisberger, "Observation of nonexponential magnetic penetration profiles in the Meissner state: A manifestation of nonlocal effects in superconductors," Phys. Rev. B 72, 024506 (2005).

${ }^{8}$ T. Prokscha, K. H. Chow, Z. Salman, E. Stilp, and A. Suter, "Direct observation of hole carrier density profiles and its light induced manipulation at the surface of Ge," arXiv:1909.11688 [cond-mat.mtrl-sci] (2019).

${ }^{9}$ H. V. Alberto, R. C. Vilão, R. B. L. Vieira, J. M. Gil, A. Weidinger, M. G. Sousa, J. P. Teixeira, A. F. da Cunha, J. P. Leitão, P. M. P. Salomé, P. A. Fernandes, T. Törndahl, T. Prokscha, A. Suter, and Z. Salman, "Slow-muon study of quaternary solar-cell materials: Single layers and $p-n$ junctions," Phys. Rev. Mater. 2, 025402 (2018).

${ }^{10}$ J. Woerle, T. Prokscha, A. Hallén, and U. Grossner, "Interaction of low-energy muons with defect profiles in proton-irradiated Si and $4 \mathrm{H}-\mathrm{SiC}$," Phys. Rev. B 100 , 115202 (2019).

${ }^{11}$ T. Prokscha, E. Morenzoni, K. Deiters, F. Foroughi, D. George, R. Kobler, A. Suter, and V. Vrankovic, "The new beam at PSI: A hybrid-type large acceptance channel for the generation of a high intensity surface-muon beam," Nucl. Instrum. Methods Phys. Res., Sect. A 595, 317-331 (2008).

${ }^{12}$ J. Gil, H. Alberto, R. Vilão, J. P. Duarte, P. Mendes, N. A. de Campos, A. Weidinger, C. Niedermayer, M. Yakushev, R. Pilkington, R. Tomlinson, and S. Cox, "High temperature trapping of muons in $\mathrm{CuInSe}_{2}$ and $\mathrm{CuInS}_{2}$," Physica B 289-290, 567-569 (2000)

${ }^{13}$ R. C. Vilão, J. M. Gil, H. V. Alberto, J. Duarte, N. A. de Campos, A. Weidinger, M. Yakushev, and S. Cox, "Muon diffusion and trapping in chalcopyrite semiconductors," Physica B 326, 181-184 (2003).

${ }^{14}$ R. C. Vilão, H. V. Alberto, J. M. Gil, J. P. P. Duarte, N. A. de Campos, A. Weidinger, and M. Yakushev, "Hydrogen states in $\mathrm{CuInSe}_{2}-\mathrm{A} \mu \mathrm{SR}$ study," Physica B 340-342, 965-968 (2003), proceedings of the 22nd International Conference on Defects in Semiconductors. 
${ }^{15}$ H. V. Alberto, R. C. Vilão, J. M. Gil, J. Piroto Duarte, R. B. L. Vieira, A. Weidinger, J. P. Leitão, A. F. da Cunha, M. Sousa, J. P. Teixeira, P. A. Fernandes, P. M. P. Salomé, K. Timmo, M. Loorits, A. Amato, H. H. Luetkens, T. Prokscha, A. Suter, and Z. Salman, "Muonium states in $\mathrm{Cu}_{2} \mathrm{ZnSnS}_{4}$ solar cell material," J. Phys.: Conf. Ser. 551, 012045 (2014).

${ }^{16}$ R. C. Vilão, H. V. Alberto, J. M. Gil, and A. Weidinger, "Thermal spike in muon implantation," Phys. Rev. B 99, 195206 (2019).

${ }^{17}$ R. C. Vilão, R. B. L. Vieira, H. V. Alberto, J. M. Gil, and A. Weidinger, "Role of the transition state in muon implantation," Phys. Rev. B 96, 195205 (2017).

${ }^{18}$ R. C. Vilão, R. B. L. Vieira, H. V. Alberto, J. M. Gil, A. Weidinger, R. L. Lichti, P. W. Mengyan, B. B. Baker, and J. S. Lord, "Barrier model in muon implantation and application to $\mathrm{Lu}_{2} \mathrm{O}_{3}$," Phys. Rev. B 98, 115201 (2018).

${ }^{19}$ H. V. Alberto, A. Weidinger, R. C. Vilão, J. P. Duarte, J. M. Gil, N. A. de Campos, J. S. Lord, and S. F. J. Cox, "Muonium as a probe of electron spin polarisation in CdTe," Physica B 404, 5110-5112 (2009).

${ }^{20}$ H. V. Alberto, A. Weidinger, R. C. Vilão, J. P. Duarte, J. M. Gil, J. S. Lord, and S. F. J. Cox, "Mechanisms of electron polarization of shallow muonium in CdTe and CdS," Phys. Rev. B 81, 245205 (2010).

${ }^{21}$ H. V. Alberto, R. C. Vilão, J. P. Duarte, J. M. Gil, A. Weidinger, J. S. Lord, and S. F. J. Cox, "Electron polarization and formation probability of bound muonium in CdS and Si," Phys. Rev. B 86, 035203 (2012).

${ }^{22}$ G. Allodi, FMINUIT-A binding to Minuit for Matlab, Octave and Scilab, 2010, http://www.fis.unipr.it/ giuseppe.allodi/Fminuit/Fminuit_intro.html \#Acknowledgments; accessed 9 June 2019.
${ }^{23}$ S. Kreitzman, R. Kiefl, D. Noakes, and J. Brewer, "Thermally activated muonium formation in $\mathrm{Al}_{2} \mathrm{O}_{3}$ and $\mathrm{BaF}_{2}$," Hyperfine Interact. 32, 521 (1986).

${ }^{24}$ V. Storchak, J. H. Brewer, and G. D. Morris, "Quantum transport of electronic polarons in sapphire," Phys. Rev. B 56, 55-58 (1997).

${ }^{25}$ P. M. P. Salomé, R. Ribeiro-Andrade, J. P. Teixeira, J. Keller, T. Törndahl, N. Nicoara, M. Edoff, J. C. González, J. P. Leitao, and S. Sadewasser, "Cd and Cu interdiffusion in $\mathrm{Cu}(\mathrm{In}, \mathrm{Ga}) \mathrm{Se}_{2} / \mathrm{CdS}$ hetero-interfaces," IEEE J. Photovoltaics $\mathbf{7}$, 858-863 (2017).

${ }^{26}$ E. Ribeiro, H. V. Alberto, R. C. Vilão, J. M. Gil, A. Weidinger, P. M. P. Salomé, T. Prokscha, A. Suter, and Z. Salman, "CdS versus ZnSnO buffer layers for a CIGS solar cell: A depth-resolved analysis using the muon probe," EPJ Web Conf. (to be published).

${ }^{27}$ D. Liao and A. Rockett, "Cu depletion at the CuInSe 2 surface," Appl. Phys. Lett. 82, 2829-2831 (2003)

${ }^{28}$ Y. Yan, K. M. Jones, J. Abushama, M. Young, S. Asher, M. M. Al-Jassim, and R. Noufi, "Microstructure of surface layers in $\mathrm{Cu}(\mathrm{In}, \mathrm{Ga}) \mathrm{Se}_{2}$ thin films," Appl, Phys. Lett. 81, 1008-1010 (2002).

${ }^{29}$ R. Herberholz, U. Rau, H. W. Schock, T. Haalboom, T. Gödecke, F. Ernst, C. Beilharz, K. W. Benz, and D. Cahen, "Phase segregation, Cu migration and junction formation in $\mathrm{Cu}(\mathrm{In}, \mathrm{Ga}) \mathrm{Se}_{2}$," Eur. Phys. J.: Appl. Phys. 6, 131-139 (1999).

${ }^{30}$ P. M. P. Salomé, J. Keller, T. Törndahl, J. P. Teixeira, N. Nicoara, R. Andrade, D. G. Stroppa, J. C. González, M. Edoff, J. P. Leitão, and S. Sadewasser, "CdS and $\mathrm{Zn}_{1-x} \mathrm{Sn}_{x} \mathrm{O}_{y}$ buffer layers for CIGS solar cells," Sol. Energy Mater. Sol. Cells 159 272-281 (2017). 